\title{
Transdiagnostic group therapy for people with self- critic and low self esteem, based on compassion focused therapy principles
}

\author{
Bodil Andersen* ${ }^{*}$ and Peter Hørslev Rasmussen
}

\begin{abstract}
Background: People with mental health problems often have difficulties linked to high self-criticism and shame, and may be fearful of and resistant to compassionate and prosocial motives. Compassion focused therapy (CFT) is specifically designed to help people address these difficulties, cultivate and build compassionate motives and emotions for self and others. There is increasing evidence for the effectiveness of CFT for both individuals and groups. This study, describing a protocol and evaluating a protocol-based CFT group treatment, adds to the evidence base in a Danish community transdiagnostic group of patients, suffering from shame, self- criticism, depression and anxiety.
\end{abstract}

Methods: This is a naturalistic study from a private psychiatric practice. Based on CFT theory and practice we developed a protocol for group therapy. As part of a routine procedure in the clinic, people were rated according to BAI, BDI and SES pre-and post-treatment. Ten groups, involving 102 patients with various psychiatric diagnosis followed a ten- week compassion mind- training programme with an eleven ${ }^{\text {th }}$ follow-up session.

Results: CFT Group treatment significantly reduced the group participants' symptoms of depression and anxiety while increasing self-esteem. There was no significant difference in outcome between the various diagnostic categories.

Conclusions: For this cohort of patients with severe psychological problems, protocol-based compassion focused group therapy was an effective treatment regardless of their diagnosis.

Keywords: Manual, Trans-diagnostic group therapy, Compassion focused therapy , Low self-esteem , Self-criticism, Affective disorder, Anxiety disorder, Personality disorder

\section{Background}

Diagnosis specific protocols are developed for a variety of different psychiatric conditions [1], but it is very timeconsuming and sometimes difficult to gather people for diagnosis specific groups. This is problematic, because a psychiatric practise, at least in Denmark, faces the challenge of having to treat a relatively large number of patients with an array of different psychiatric diagnoses within a reasonable time. However, working with and comparing disorder-specific models and protocols reveals that patients across diagnosis share common underlying processes. Harvey et al. [2] synthesized

\footnotetext{
*Correspondence: bodil.andersen@dadlnet.dk

Kognitiv Center Fyn, Odense, Denmark
}

emerging evidence for these commonalities and pointed out the following cognitive and behavioural processes: Selective attention, explicit and implicit selective memory, a wide range of reasoning processes, recurrent thinking (i.e., rumination and worry, avoidance and safety-seeking behaviours).

In a group- therapeutic setting for patients with a wide spectrum of psychiatric diagnosis, it is therefore very relevant to focus the attention on transdiagnostic aspects or patterns. There is evidence $[3,4]$ supporting the advantage of addressing these patterns specifically. Mansell et al. [5] state that it "enables a flexible treatment approach that can be applied across a range of presentations, including comorbidity thus producing more 
potent interventions that better address comorbidity". The most frequent pattern is the tendency to ruminate. Studies have demonstrated that ruminative thinking predicts depression over and above its shared variance with several types of negative cognitions [6,7]. Wells and Papageorgiou [8] proposed that rumination specifically encompasses self-focused thinking, and negative appraisals of the self, emotions, behaviours, situations, life stressors, and coping. The impact of rumination may be sadness, anxiety, worry. The rumination itself may strengthen and maintain depression and anxiety [6]. Various therapies are developed in order to specifically address the rumination (i.e. MBCT, Metacognitive Therapy, and Rumination Focused Cognitive Therapy).

A considerable number of patients show ruminations of a very self-critical nature. Self-criticism is significantly associated with shame-proneness and both permeate many disorders, increase vulnerability, effect expression of symptoms and elevate risk of relapse $[9,10,11]$. The pathogenic qualities of shame and self- criticism link to two key processes. The first is the degree of selfdirected hostility, contempt and self- loathing. The second is the relative inability to generate feelings of self-directed warmth, soothing and self- liking [10]. Compassion, however, seems to be able to transform experiences of distress, according to Bates [12] by reducing felt sense of being alone, by softening of adverse reactions to pain, by dis-identification with symptoms, by arousal of alternative affect, by creating spaciousness around problems, and by opposing the essence of social anxiety. Compassionate mind training is highly suitable for people with high shame and self- criticism. The CFT perspective, among other things, emphasises common human conditions by incorporating elements of evolutionary theory, neuropsychology/ biology and which focuses more intensely on developmental psychology. [13-18].

Many years of experience with CBT and MBCT has shown the authors that these approaches embody numerous therapeutic possibilities for helping patients with a wide variety of mental health conditions. However, struggles with issues like shame, guilt and self-criticism seem to challenge some groups of patients to such an extent as to render them unable to benefit sufficiently from CBT. Many patients experience this as a so called "heart and head lack", and thus have no emotional impact of cognitive restructuring. Their therapeutic insight has no immediate effect on an emotional and behavioural level. This has underscored the need for a therapy that more explicitly enables us to work with the barriers mentioned above.

There are many advantages for choosing to work with CFT in a group therapy context, as CFT highlights the significance of the relational aspects, common human conditions, and the importance of being able to give and receive compassion $[19,20]$.
There is increased but limited research on the effectiveness of group-based CFT for people with chronic health problems. Gilbert and Procter [10] did a 12 week pilot study for people with high shame and selfcriticism, Lucre and Corten [20] did a 16-week group therapy for people with personality disorders and found significant reductions in shame measures and social comparison, Braeler et al. [21] conducted the first RCT of CFT 16-weeks group therapy for people with psychosis. Findings supported the feasibility of group CFT in psychosis, reducing depression in particular. CFT was associated with no adverse effects, high acceptability and great clinical improvement.

In CFT, the focus is traditionally on the therapeutic process, and a manual-based approach is therefore unc ommon. However, Braeler [21] developed a protocol for treatment of psychosis, based on a forensic group manual [22], generic CFT [23], aspects of mindfulness [24], and group processes in psychosis [25]. Lucre [20] advised three- main components to the groupwork process: formulation and psychoeducation, compassionate mind training, and planning for practice, but not a protocol. Bates [11] used a CBT protocol for social anxiety with compassion elements. In the Gilbert and Procter study [10] the group-based format was highly task- focused and unfolds in a series of steps, very much in collaboration with the participants. Van den Brink, E. \& Koster, F. [26] developed an eight- weeks training program, called Mindfulness-Based Compassionate Living, which is both a training program for therapists and a treatment guide.

\section{Aims}

This study aims to develop and test a treatment protocol based on CFT principles. Furthermore to investigate whether CFT could work as a short-term group intervention, thereby enabling us to offer the programme to more patients within a manageable timeframe.

\section{Method}

This is a naturalistic study, reporting basic data obtained in the clinic as routine procedure. The patients have given their consent to participate in group treatment based on CFT principles. As a standard procedure, treatment was evaluated using standard measures. Prior to start patients received oral and written information. This study was a register study using data obtained as part of the standard treatment.

The group size was 10-12 participants. This size was selected to ensure the group's viability and to create the best conditions for the therapeutic process. The reason for being two therapists throughout the programme was the possibility of discussing group processes and creating dynamic sessions by switching therapist during sessions. 
Additionally one therapist would be able to leave the group for a short while if a participant felt unwell and had to withdraw.

Each group had 10 weekly sessions plus a follow-up session.

\section{Measures}

The inventories used are standard measures in CBT treatment.

\section{Beck depression inventory}

The BDI is a familiar, 21-item scale for measuring depression in clinical populations. Beck et al. [27-29] provided a major review of the psychometric properties, and outlined various recommendations and guidelines regarding the use of the BDI.

\section{Beck anxiety inventory}

Beck Anxiety Inventory (BAI) [30] is a 21-question selfreport inventory. It measures the severity of physical anxiety symptoms. Final scores can fall into the Normal, Borderline, Clinical, Mild, Moderate, Severe, Extreme range for anxiety.

\section{Rosenberg self-esteem scale (RSE)}

The RSE [31] is a widely used 10-item measure of selfesteem (e.g., "I take a positive attitude towards myself"). It has high test-retest reliability and good internal consistency [32].

\section{Procedure}

The psychiatrist assessed and diagnosed every participant, using ICD10 criteria, prior to treatment. The assessment consisted of a thorough examination of the course of illness together with a specific focus on early life events; and specifically self- critical statements and shame- based memories. If the psychiatrist noted high levels of shame and self-critic, the patient was found suitable for the CFT-group and received an oral introduction of the CFT treatment paradigm. If the patient accepted, she referred him or her to group therapy after one or more consultations. A large proportion of the participants had previously received various forms of psychiatric and/or psychological treatment.

The exclusion criteria coincided with the more traditional, general criteria often used for group therapy. Examples of such criteria were active abuse, active psychosis and acting out behaviour.

The patients consecutively signed up for group therapy. Therapy was launched when a group-size of 10-12 participants was reached.

In the first and last session, patients rated themselves using Beck Depression Inventory (BDI), Beck Anxiety Inventory (BAI) and Rosenberg Self-Esteem Scale (RSE).
Each group course consisted of 10 weekly two-hour sessions with a break halfway through, and after one or two months an additional follow-up session.

\section{The treatment protocol}

The overall structure of sessions two to ten is as follows:

- Meditation/ exercise

- Discussion/review of previous session

- Discussion/review of homework

- Theme of the day

- Meditation/ exercise

- Discussion/review of new homework

\section{Content of individual sessions \\ First session}

The group settles and there is a mutual introduction, including presentation, group purpose, and group rules. After exploring peoples' hopes, fears and motivation for being in the group, the therapists present the fundamental ideas of CFT. This includes the new brain/old brain problem, common humanity, the importance of attachment, and the fulfilment of basic needs from the perspective of psychological development. The therapists outline The three affect regulation systems [33], highlighting the importance of anxiety-reducing safety strategies. Lastly, grounding is established through mindfulness meditation in the form of Soothing Breathing Rhythm. [34]. The participants discuss, in pairs, possible obstacles to doing the homework, which consists of a daily soothing breathing exercise guided by audio file. It is important to acknowledge Fears, Blocks and Resistance arising in the meeting with compassion and address these emotions /reactions already from the early stages of treatment.

\section{Second session}

The group settles. There is a grounding exercise using Soothing Breathing Rhythm. The group discusses anxiety, fears, and worries from a psychological and biological perspective. Illustrations outline the mechanisms of anxiety. There is a focus on the prefrontal cortex, the limbic system and the role of different brain structures (i.e. the amygdala and hippocampus). The therapists highlight the similarity between the physical reactions associated with experienced threats, and imagined threats. Conditioning is mentioned as one possible psychological factor in the development of anxiety. After a break, a new exercise - Safe Place [35] is introduced. The aim is to give the people the sense that inner images of a soothing nature can have an immediate physical and emotional effect. Some might find it challenging or impossible to create such images. Therefore, it is important 
to adopt a 'playful' rather than performance-oriented approach to the exercise.

Finally, the group reflects on the human motivation system, distinguishing between motives and emotions categorized as threat-focused, drive / reward-focused and soothing/contentment-focused. The affect systems constitutes a preliminary case formulation. People reflect on how they experience the balance between the affect systems. They present specific situations that influence the individual systems. Homework is to meditate doing the soothing breathing rhythm and safe place exercises and to make a note of situations where the threat protection system, the drive system and the soothing system, respectively, is predominating.

\section{Third session}

After settling, the session is set about with the exercise Safe Place with Stone. The stone is a conditioning object. The aim is to enable people, over time, to get into contact with their safe place by touching the stone. They share experiences of visualising their safe places and of overcoming the obstacles that some may encounter during the exercise. There is a focus on the participants' experiences from their homework dealing with the affect systems. They reflect on their discoveries and experiences, outlining specific factors that affects the balance between the systems. Besides situations associated with the drive and safeness systems participants identify trigger situations affecting the threat system. The Compassion model [36] is introduced during the second part of the session, firstly by running through the theory in dialogue with the group, emphasising the complexity of the concept of compassion, both attributes and skills. Secondly, the exercise - The Compassionate Self [37] is presented. The purpose is to encourage an experiencebased understanding, using techniques from method acting. During this exercise, the participants create an image of themselves at their compassionate best. The focus is on developing a compassionate attention, thinking, feeling and behaviour linked to the affiliative soothing system. Finally, participants use their own examples of trigger situations as a basis for working compassionately with their negative and self-critical appraisals. Exercising the compassionate self and working compassionately with trigger situations are the participants' homework.

\section{Fourth session}

After settling and Compassionate Self Meditation, the focus is mainly on peoples' work with trigger situations. Using personal experiences, the group rehearse compassionate reappraisal [30] of negative thoughts associated with trigger situations. They do not engage in challenging self-criticism directly. During this exercise, it is vital to demonstrate the difference between a compassionate approach and for instance a more rational and reserved response. Some participants may not find their reappraisals soothing or helpful, perhaps because these are self-critical in content and tone. Some may experience fears and blocks towards compassion. It is very vital to notice and address these issues in the group. The homework assignment is to identify trigger situation and rehearsing a compassionate approach to negative thoughts and emotions.

\section{Fifth session}

Besides the regular elements of settling, meditation and homework, the emphasis is exercising compassionate letter-writing [38]. The group splits up into smaller groups, and people are asked to read a not particularly compassionate letter. Using their experience from the previous sessions, they draw up a more compassionate draft. They share their drafts with the whole group. Again, vital elements of compassion are highlighted, not least the compassionate tone of voice. As well as meditation, the participants' homework consists of writing a compassionate letter to themselves.

\section{Sixth session}

After settling and meditation, the group summarises homework, i.e. the compassionate letter writing. People are encouraged to read their compassionate letters to themselves aloud to the group. In some cases, one of the therapists may read the letter at the participant's request. Again, the tone of the letter, both in writing and reading is clearly crucial to the emotional and physical reactions experienced [39]. The therapists invite people to express their emotional reactions to the letters read aloud, and call explicitly for their compassionate feedback. After the break, people try to identify external sources of selfcritical thoughts, and in this connection, the idea / concept of the critical self/ the inner critic is introduced [40]. The fundamental assumption is that no one is born self-critical and that self-criticism will always come from external, experience-based sources. People share their individual reflections on the external sources that shaped their inner critics, for example parents, siblings, teachers, and classmates. During the last part of the session, the group considers the function of the inner critic, it's advantages, as well as it's disadvantages. They all engage in the exercise The Good Teacher and the Bad Teacher, by giving examples of qualities associated with a good and a bad teacher respectively. The aim is to make out whether the self-critical stance is helpful or not. In addition to meditation, the participants' homework is to continue working with the sources of selfcritical thoughts. 


\section{Seventh session}

Following the regular elements, including settling, meditation, and review of homework, the group is introduced to the Threat/Safety Strategies Formulation [41]. Using a pre-printed example of a woman with a rather traumatic background, the group discusses how the past has affected her, with regard to her schemes and key fears. The group identifies her possible safety strategies and discusses the subsequent positive and negative consequences. A case formulation can only embody part of a person's story, and no-one's story can fit in a sheet of A4 paper. It is important to point this out. The goal of creating a case formulation is to give people a chance to gain greater insight into the background for and the possible unintentional consequences of their safety behaviour. This includes viewing safety behaviour as an entirely natural and probable response to traumatic childhood experiences and the subsequent fear of rejection and exclusion. In the last part of the session, the participants work individually on their own case formulation and complete it as part of their homework. During case formulation work, participants may experience an entirely understandable grief at the conditions to which they have been exposed and the consequences of these conditions. It is important to see, acknowledge, and handle these reactions appropriately and compassionately.

\section{Eighth session}

After settling, meditation and homework review, the focus is on reviewing peoples' experiences with their case formulation homework. Some will be willing to share their formulation with the group. Again, the other participants' compassionate responses to the person presenting a case formulation are of very great importance. Some may identify with the case- story; others may react emotionally to the often very harsh and austere life stories. Working with case formulation proves to be a highly effective illustration of the fact that the common human condition entails a life with challenges and suffering, albeit to various degrees. Using Paul Gilbert's expression 'it's not your fault but it's your responsibility to change it' and the personal case formulations as a basis, people are encouraged to articulate a more compassionate mind set: "How would I like my interpersonal relations to be?", "What would be a more compassionate view of myself, others and the world?" "Can I hold an image of this in my mind's eye?" "What new rules for living and strategies would be most appropriate in that connection?" "How can I build up my motivation and my strength in order to pursue the new goals?" In addition to meditation, homework is to focus on and practise the new strategies.

\section{Ninth session}

Following settling, meditation and homework review, the main theme of the session is the construction of $a$ Compassionate Image [42]. As well as people can have inner mental images of a threatening nature, they can also create mental images of a compassionate nature. People acknowledge that the qualities of the new brain - the ability to create soothing mental images, for instance - will reinforce the safeness-soothing system, thereby reducing anxiety and worry. Firstly, people reflect on which traits and sensory qualities they individually associate with soothing and safety. They then describe in writing their own Compassionate image, which could be, for example, a person or animal that has these sensory qualities (i.e. a specific scent, colour, appearance, texture and voice). The compassionate image should also possess strength, wisdom and courage together with a deep commitment to enhance the person's wellbeing. The session closes with a meditation during which the Compassionate Image is visualised. Some may find it hard to imagine a compassionate image during the first try. It is therefore important to stress that this is a process fostered by a playful approach.

The homework consists of meditation incorporating a Compassionate Image. The participants are also encouraged to use the Compassionate Image as a discussion partner for tasks such as compassionate letter-writing and compassionate reappraisal when in trigger situations.

\section{Tenth session}

After meditation and homework review, the participants work with Fears, Blocs and Resistance to compassion [43]. Therapists have addressed this topic on an ad-hoc basis in the other sessions, but this session focuses explicitly on this issue. As well as working together in a group, the participants reflect in pairs on potential barriers and possible ways of overcoming them. The group then makes up a list of behavioural initiatives people could launch in the future to stimulate their safeness system, as well as discusses the possible barriers and ways of overcoming these.

Exercise using a compassionate Image follows the break, and the participants then reflect on $\mathrm{f}$ their take home messages from the group sessions and share them in plenum. Loving-kindness meditation (metta) [44] and final statements about the group course from all group members conclude the last session.

\section{Follow-up (11th session)}

The follow-up session begins with a meditation, after which the group engages in a plenary discussion; each group member describes his or her experience of working with compassion during the relatively long period since last session. People point out specific barriers and 
obstacles. The group has an opportunity to repeat exercises and meditations from various sessions. At the end of the session, participants individually reflect on how they could each hold onto a compassionate perspective in the future.

\section{Results}

\section{Participants}

The study comprised 102 patients with various psychiatric diagnosis, referred to psychiatrist in private practice by their GPs.

\section{Drop outs}

There were fifteen drop outs and missing end data on twelve patients, who completed treatment. This gives a dropout rate on $14.7 \%$, which is to be expected [45]. The reason for dropping out is not clear in all cases. Some stated that the anxiety of being in a group was too high. Some did not feel connected to the group, and some left due to other external circumstances. There is no difference between dropouts and missing data patients, compared to the rest with respect to the pre-ratings.

\section{Diagnostic grouping}

The participants split up into the following diagnostic categories: $36 \%$ have an affective disorder, 35\% an anxiety disorder, and $29 \%$ a personality disorder. Of the total number of patients, $28 \%$ meet the criteria for both an axis 1 and an axis 2 diagnosis.

\section{Statistics}

Data material consists of observations from 75 participants, from 10 groups (Table 1). Three central response variables were measured before and after treatment. Explanatory

Table 1 Sociodemographics

\begin{tabular}{llll}
\hline & Male & Female & Total (percent of total) \\
\hline Number (\% of total) & $12(16 \%)$ & $63(84 \%)$ & 75 \\
Age 20-29 & 1 & 6 & $7(9)$ \\
Age 30-39 & 2 & 23 & $25(33)$ \\
Age 40-49 & 9 & 15 & $24(32)$ \\
Age 50-59 & 0 & 12 & $12(16)$ \\
Age 60-69 & 0 & 7 & $7(9)$ \\
Co-habitating & 4 & 28 & $32(43)$ \\
Parent & 5 & 41 & $46(61)$ \\
Employed & 6 & 21 & $27(36)$ \\
No qualifying education & 2 & 8 & $10(13)$ \\
Short tertiary education & 1 & 9 & $10(13)$ \\
Bachelor or equivalent & 3 & 28 & $31(41)$ \\
Master or higher education & 6 & 10 & $16(21)$ \\
\hline
\end{tabular}

The numbers in the columns specify the number of subjects in each category, numbers in parentheses specify percentage of total variables are socio-economic conditions (Table 1), psychiatric diagnosis and adverse childhood experience (Table 2). Distribution of BDI and BAI delta values is normal, and the $p$ values in a linear regression analysis therefore true. The delta SES show a positive skew, which can have an impact on the precision of the $\mathrm{p}$ values.

A multiple regression analysis shows that neither age nor gender (Table 3) nor diagnosis have significant effect on treatment outcome. The socio-economic variables, added in a similar analysis, show a positive effect of labour market affiliation and BDI reduction, and a SES rise when living with a partner.

\section{Sample description}

Forty-three\% live with a partner and have in average one child. Thirty-six \% have an affiliation to the labour market. The majority has an education of medium length or a higher education. Forty-one \% experienced parents' divorce, thirty-one \% have been subjected to ill-treatment, sixteen \% to sexual abuse, twenty-four \% to bullying, twenty \% experienced bereavement, and forty-one \% grew up with one or two physically or mentally ill parents (Table 2).

\section{Outcome related to diagnosis}

As shown in Fig. 1 below, the depression and anxiety score for the cohort as a whole fell while the self-esteem score rose.

From a transdiagnostic perspective it is relevant to consider the extent to which outcomes are comparable across the diagnoses. We therefore chose to look at the effect of the treatment in relation to the separate diagnostic categories.

\section{Depression score measured by BDI}

There was a significant change in depression score for all diagnostic groups, and there was no significant difference between the groups (Fig. 2).

Legend: The box designates the median and the upper and lower quartiles. Whiskers/lines represent the max.

Table 2 Childhood experiences

\begin{tabular}{llll}
\hline & Male & Female & Total (percent of 75 patients) \\
\hline Divorce & 3 & 28 & $31(41)$ \\
Violence & 4 & 17 & $21(28)$ \\
Incest & 2 & 10 & $12(16)$ \\
Substance abuse & 2 & 15 & $17(22)$ \\
Bullying & 4 & 14 & $18(24)$ \\
Death & 2 & 13 & $15(20)$ \\
III parents & 4 & 27 & $31(41)$ \\
\hline
\end{tabular}

This table only includes the 75 subjects who completed the treatment The numbers in the columns specify the number of subjects in each category, numbers in parentheses specify percentage of total 
Table 3 Multiple regression with early life stressors as explanatory

\begin{tabular}{|c|c|c|c|}
\hline & $\begin{array}{l}\text { Change in } \mathrm{BDI} \\
\mathrm{BDI} p \text {-value } \\
\text { (95\% coefficient Cl) }\end{array}$ & $\begin{array}{l}\text { Change in BAl } \\
p \text {-value } \\
\text { ( } 95 \% \text { coefficient Cl) }\end{array}$ & $\begin{array}{l}\text { Change in SES } \\
\text { p-value } \\
\text { (95\% coefficient Cl) }\end{array}$ \\
\hline BDI (pre) & $\begin{array}{l}p=0.034 \\
(-0.51-0.02)\end{array}$ & $\mathrm{N} / \mathrm{A}$ & N/A \\
\hline BAI (pre) & N/A & $\begin{array}{l}p=0.000 \\
(0.77-0.32)\end{array}$ & N/A \\
\hline SES (pre) & N/A & $\mathrm{N} / \mathrm{A}$ & $\begin{array}{l}p=0.017 \\
(-0.58-0.057)\end{array}$ \\
\hline $\begin{array}{l}3 \text { or } \\
\text { more } \\
\text { early life } \\
\text { stressor }\end{array}$ & $p=0.538$ & $p=0.744$ & $p=0.780$ \\
\hline Male & $p=0.711$ & $p=0.278$ & $p=0781$ \\
\hline Age & $p=0.761$ & $p=0.770$ & $p=0465$ \\
\hline
\end{tabular}

BDI Beck's Depression Index; BAI; SES; CI Confidence interval

and min. Observations within the range of the 1 . and 3 . quartiles $+/-1.5 * \mathrm{IQR}$ (InterQuartile Range). Dots are outlier cases. Bar plots show change from pre to post measurements for the response variables. BDI; Becks depression index.

\section{Anxiety score measured by BAI}

Figure 3 also shows a significant reduction in anxiety scores for all three diagnostic categories. The difference is not significant when the three groups are compared. However, the greatest effect tends to be in the group of patients who meet the anxiety diagnosis criteria.

Legend: The box designates the median and the upper and lower quartiles. Whiskers/lines represent the max. and min. Observations within the range of the 1 . and 3. quartiles +/- 1.5*IQR (InterQuartile Range). Dots are outlier cases. Bar plots show change from pre to post measurements for the response variables. BAI; Becks anxiety index.

\section{Self-esteem score measured by SES}

Figure 4 shows a significant increase in SES score, indicating an increase in self-esteem. However, the greatest effect tends to be in the group of patients who meet the criteria for a personality disorder diagnosis.

Legend: The box designates the median and the upper and lower quartiles. Whiskers/lines represent the max. and min. Observations within the range of the 1 . and 3 . quartiles +/- 1.5*IQR (InterQuartile Range). Dots are outlier cases. Bar plots show change from pre to post measurements for the response variables. SES; Rosenbergs self-esteem score.

It is relevant to consider the treatment effect size.

The conclusion, as shown in Table 4 is that the treatment has a moderate to large effect on BDI and SES and a moderate effect on BAI.

\section{Socio-economic factors}

Table 3 shows that the greater the pre scores of BDI and BAI, and the smaller the pre score of SES the greater the treatment response, indicating that people with severe depressive and anxiety symptoms and low self-esteem profited the most from treatment .

The extend of childhood adversities did not seem to have an impact on treatment, neither did socioeconomic factors.

\section{Discussion}

\section{On group treatment}

Each group had 10 weekly sessions and a follow up session. This is short time treatment, which mirrors the

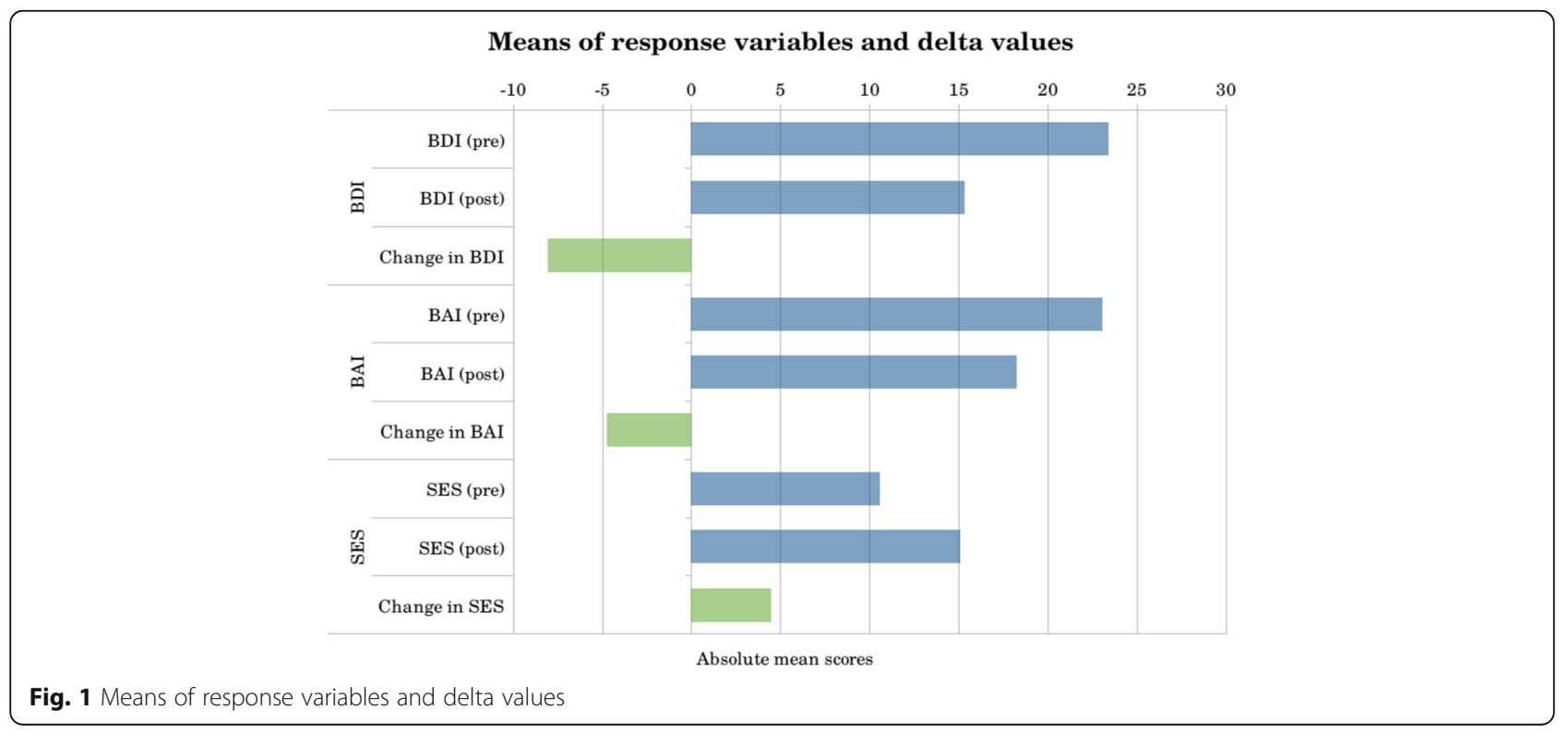




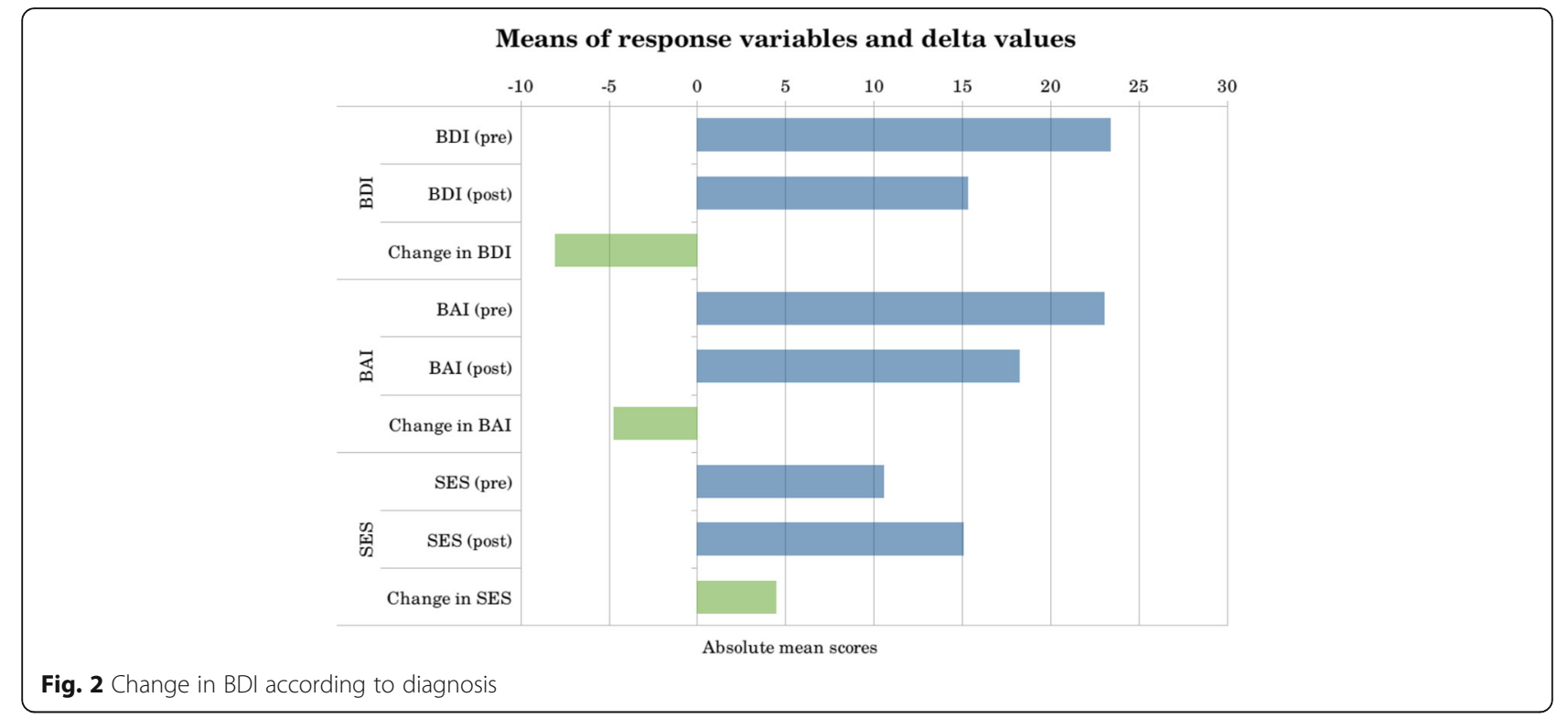

demands from the Danish health service. Our results seem to indicate that there is a treatment effect in 10 sessions. The impact is, however, that there is a risk of forcing the therapeutic process.

A transdiagnostic approach makes it possible to gather a sufficient number of participants within a manageable period to be able to initiate a group course. A big advantage is that this shortens the waiting time for patients.

\section{On using a manual}

Paul Gilbert among others has accentuated that CFT is a collaborative, step by step process, agreed by patient and therapist as one proceeds, and it is important not to impose [10]. It is therefore somewhat controversial in a CFT context to use a protocol. So why use a protocol?

There are several reasons: It provides therapist with guidelines and some directions in therapy. It ensures that CFT key issues are addressed, and a certain order regarding the various CFT elements is obtained. Finally It facilitates research in various ways: It is possible to establish and compare different groups, it is possible to investigate which elements of CFT are the most powerful, and finally it will be easier to ascertain, whether CFT, according to standards, has in fact been delivered. It is however, crucial that the protocol does never take precedence over the therapists' attention toward group processes, including interpersonal relations. The group

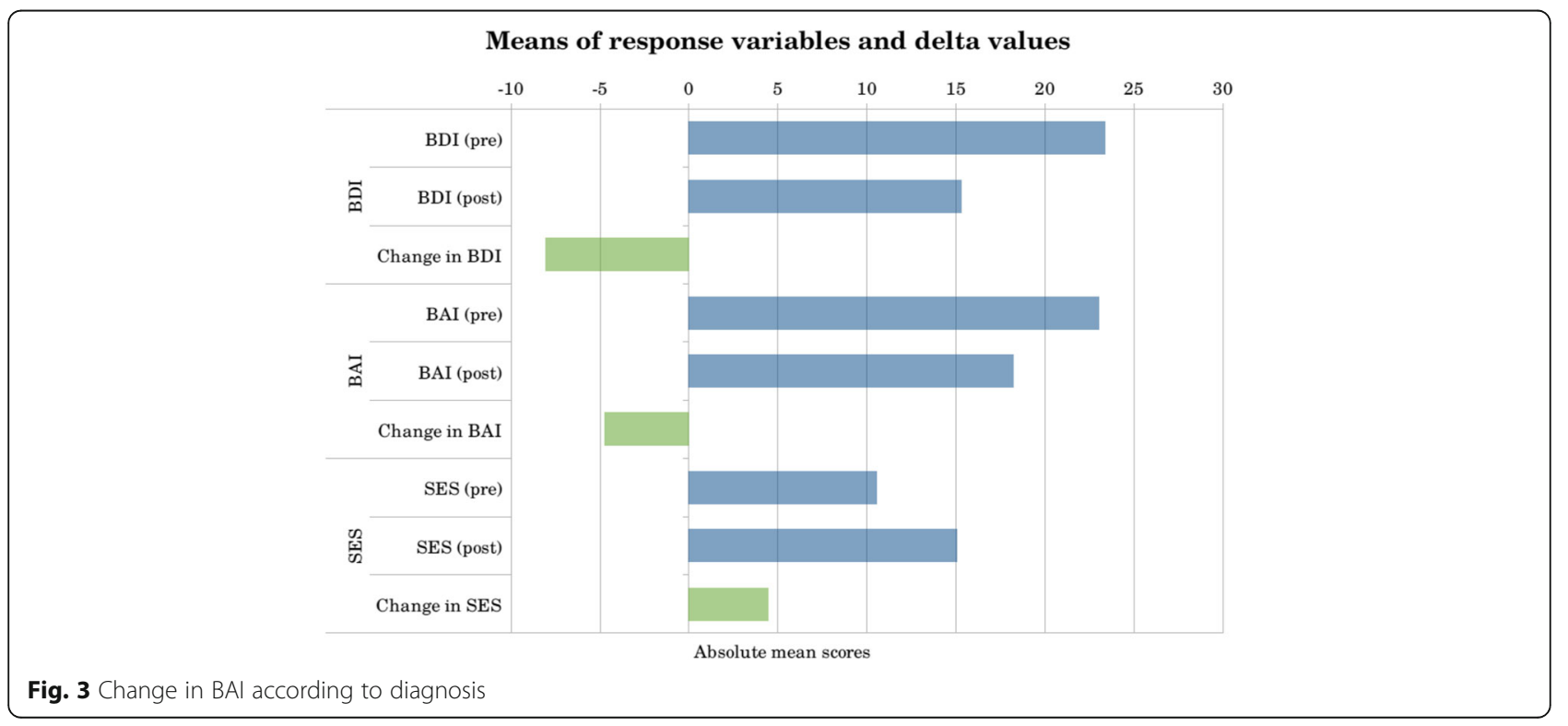




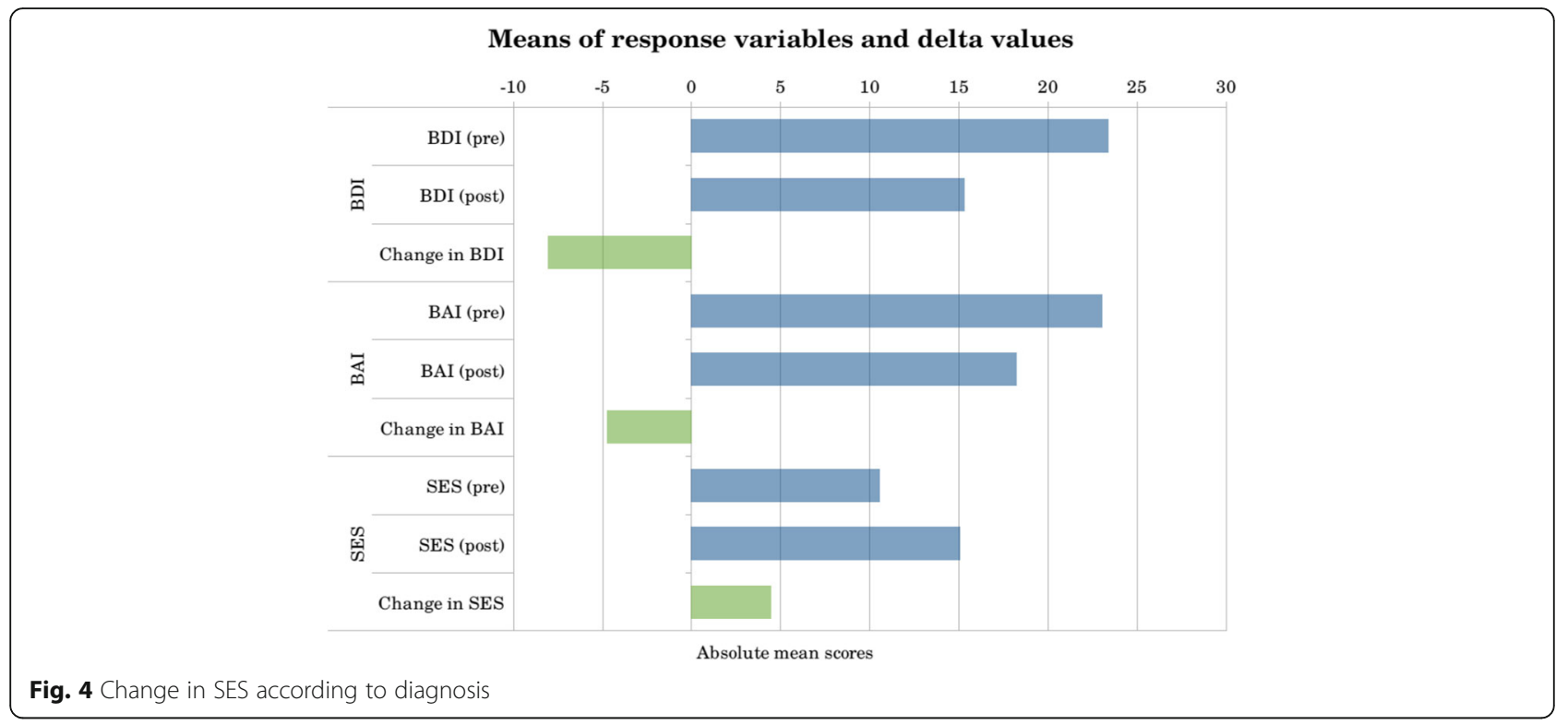

format in particular facilitates the Flow of Compassion. As Yalom [46] specifies: "cohesiveness, the sheer experience of being a valued member of a group may for some patients be the major vehicle for change. For others, cohesiveness provides the safety and support that allows one to explore oneself, to request interpersonal feedback and to experiment with new behaviour".The following statements from group members seem to support seem to support this: "It was lovely being in the group", "I could recognize so much". "I'm not alone" "I have the feeling of not being alone." "Others struggle with the same issues". "It's easy to have compassion for others and difficult to have compassion for oneself".

Given the fact that therapists act as role- models, flow of compassion between the therapists is also crucial. Therapists must be aware of that. It is therefore essential that they meet before and after the sessions, partly in order to allot tasks, partly to debrief and discuss the session, and plan next session. Bates, who applied compassion elements to CBT group therapy, underlines the flexibility required in applying the model (CBT) in order to incorporate and maximize the therapeutic benefits [12]. However, he also

Table 4 Effect sizes for the complete sample

\begin{tabular}{lllll}
\hline & Pre M (SD) & Post M (SD) & T & Effect size (Cohen's d) \\
\hline BDI & $23.35(10.71)$ & $15.31(11.81)$ & $7.94 * *$ & 0.71 \\
BAl & $22.95(11.18)$ & $18.24(10.07)$ & $3.83 * *$ & 0.44 \\
SES & $10.61(5.15)$ & $15.15(6.54)$ & $-6.70^{* *}$ & 0.75
\end{tabular}

${ }^{*} p<.05$; ** $p<.001$ BDI- Beck Depression inventory; BAl- Beck anxiety inventory; SES- Rosenberg self-esteem

Effect size $0.2=$ small effect, $0.5=$ moderate effect, $0.8=$ large effect (Cohen, 1988) mentions the risk of the model becoming lost in the complexities of group dynamics.

It is important to stress that this protocol demands well-trained CFT therapists. They must have a good understanding of engagement and -alleviation psychology, they must be able to bond and create a therapeutic relationship, and finally possess specialist CFT skills and be able to teach and practice compassion task.

\section{To compose a protocol}

We found it very important to provide group-members with a shared understanding of the therapeutic model, implying that the psycho-educative elements are primarily in the early sessions. This enhanced the feelings of safety and getting greater insight facilitated motivation for working with compassion and increased the strength and courage to do. The psycho-educative framework reduced the anxiety of being in a group and gave the group-members the possibilities of step wise self-disclosure, as well as grounding exercises (.e. meditation) helped reducing the anxiety levels. The more detailed case- formulation could easily provoke a lot of grief and sorrow and was therefore placed later in the group course, giving group members the opportunity of having trained basic self-soothing skills. This would enable them to meet the pain and grief more compassionately. Throughout the group-course there is was a natural progression in participant interaction.

We had to struggle to find a balance between the amount of psychoeducation and psychotherapy. Sometimes, one could be carried away passing on the theory with the expense of the more dynamic group processes. It was important to be aware of this risk, and leave time and space for the group to reflect. 


\section{The future perspectives}

There is a demand for RCT studies. We hope that the protocol and the very promising results will both provide a framework for group treatment, using the CFT model and encourage researchers to engage in further research of the treatment effect.

\section{Design weaknesses}

This is a naturalistic study, carried out in a private practice. It suffers from several methodological shortcomings. There is no control group and no randomization. The patient were screened by the psychiatrist, and the criteria for referral to the group was her judgement and therefore highly subjective. Twelve persons were not included in the study because of missing end data, and there was no follow- up assessment. Furthermore, there are no qualitative data, no process measures and relevant compassionate measures. We lack mediational analysis and time series analysis.

\section{Conclusion}

This study shows that it is possible in a private practice, to offer an effective, short-term treatment, using a protocol, which contains the central CFT elements. Regardless of patients' psychiatric diagnosis, the treatment significantly reduced symptoms of depression and anxiety and increased the sense of self-esteem. This clearly indicates that the treatment has transdiagnostic qualities. Using a protocol provides therapists with a good structure for the therapeutic work under due consideration for the group process. Furthermore, the protocol ensures a greater homogeneity in the group therapeutic treatment offer.

\section{Abbreviations}

BAl: Beck's Anxiety Inventory; BDI: Beck's depression inventory; CBT: Cognitive -Behavioural Therapy; CFT: Compassion focused therapy; MBCT: mindfulnessbased Cognitive therapy; SES: Rosenberg's Self-esteem Score

\section{Acknowledgements \\ First and foremost our thanks go to all the patients who followed the group sessions. We also extend grateful thanks to professor Paul Gilbert for his invaluable support and inspiration. Thanks to Anders Bo Bojesen, Jaskaran Basran and Andreas Andersen for their assistance with the statistics.}

\section{Funding}

Fonden for Faglig Udvikling af Speciallægepraksis (The Foundation for the Professional Development of Specialist Medical Practice) has provided project funding.

\section{Availability of data and materials}

The materials described in the manuscript are readily reproducible, including database and relevant data. Databases as described in the manuscript are available upon request from the researchers in a way that preserves anonymity.

\section{Authors' contributions}

Both authors have made substantial contribution to this study.

\section{Ethics approval and consent to participate}

The project has been presented to the Ethical Committee, Region of Southern Denmark, who states that it is a naturalistic study that does not need approval by the Ethical Committee. The study is approved by the Danish Data Protection Agency.

\section{Consent for publication}

Not applicable

Competing interests

The authors declare that they have no competing interests.

\section{Publisher's Note}

Springer Nature remains neutral with regard to jurisdictional claims in published maps and institutional affiliations.

Received: 7 June 2017 Accepted: 1 December 2017

Published online: 21 December 2017

\section{References}

1. Butler G, Fennell M, Hackmann A. Cognitive-behavioral therapy for anxiety disorders. Mastering clinical challenges. New York: The Guildford Press; 2008

2. Harvey A, Watkins E, Mansell W, Shafran R. Cognitive behavioural processes across psychological disorders. Oxford: Oxford University Press; 2004.

3. Barlow DH, Allen LB, Choate ML. Toward a unified treatment of emotional disorders. Behav Ther. 2004;35(2):205-30.

4. Noelen-Hoeksema S, Parker LE, Larsson J. Ruminative coping with depressed mood following loss. J Pers Soc Psychol. 1994:67(1):92-104.

5. Mansell W, Harvey A, Watkins ER, Shafran R. Cognitive behavioural processes across psychiatric diagnosis: a review of the utility and validity of the transdiagnostic approach. Int J Cogn Ther. 2008;1(3):181-91.

6. McLaughlin KA, Nolan-Hoeksema S. Rumination as a transdiagnostic factor in depression and anxiety. Behav Res Ther. 2011;49(3):186-93.

7. Spasojevic J, Alloy LB. Rumination as a common mechanism relating depressive risk factors to depression. Emotion. 2001;1(1):35-7.

8. Papageorgiou C, Wells A. Nature, functions, and beliefs about depressive rumination. In: Papageorgiou, Wells, editors. Depressive rumination. Nature, theory and treatment; 2004. p. 3-20.

9. Gilbert P, Miles JNV. Sensitivity to putdown: its relationship to perceptions of shame, social anxiety, depression, anger, and self-other blame. Personal Individ Differ. 2000:29:757-74.

10. Gilbert, P. \& Proctor, S. (2006): Compassionate mind training for people with high shame and self-criticism: overview and pilot study of a group therapy approach. Clin Psychol Psychother, 13, pp.351-379.doi:10.1002/ccp.507.

11. Neff KD. The development and validation of a scale to measure selfcompassion. Self Identity. 2003b;2:223-50.

12. Bates, T. (2005). The expression of compassion in group cognitive therapy. In Paul Gilbert's (ed): Compassion, Conceptualisations, Research and Use in Psychotherapy. UK: Routledge; pp.369-386.

13. Gilbert P. Human nature and suffering. Hove: Lawrence Erlbaum associates, Inc.; 1989.

14. Gilbert P. Social mentalities: internal 'social' conflicts and the role of inner warmth and compassion in cognitive therapy. In: Gilbert P, Bailey KG, editors. Genes on the couch: explorations in evolutionary psychotherapy. Hove: Psychology Press; 2000a. p. 118-50.

15. Gilbert P. Social mentalities: a biopsychosocial and evolutionary reflection on social relationships. In: Baldwin MW, editor. Interpersonal cognition. New York: Guilford Press; 2005b. p. 299-335.

16. Gilbert P. The compassionate mind. UK: Constable \& Robinson; 2009.

17. Cozelino L. The neuroscience of human relationships: attachment and the developing brain. New York: Norton; 2007.

18. Siegel DJ. The mindful brain: reflection and Attunement in the cultivation of well-being. New York: Norton; 2007.

19. Judge L, Cleghorn A, McEwan K, Gilbert P. An exploration of group-based compassion focused therapy for a heterogeneous range of clients presenting to a community mental health team. Int J Cogn Ther. 2012;5: 420-9. https://doi.org/10.1521/ijct.2012.5.4.420

20. Lucre, K.M. \& Corten, N. (2012): An exploration of compassion-focused therapy for personality disorders. Psychology and Psychotherapy: theory, research and Practice. Advance online publication. doi: https://doi.org/10. 1111/J.2044-8341.2012.02068.X.

21. Braehler C, Gumley A, Harper J, Wallace S, Norrie J, Gilbert P. Exploring chance processes in compassion focused therapy in psychosis: result of a 
feasibility randomized controlled trial. Br J Clin Psychol. 2012;52:199-214. https://doi.org/10.1111/bjc.12009.

22. Laithwaite H, O'Hanlon M, Collins P, Doyle P, Abraham L, Porter S. Recovery after psychosis.(RAP): a compassion focused programme for individuals residing in high security settings. Behav Cogn Psychother. 2009;37:511-26.

23. Gilbert P. Compassion focused therapy: The CBT distinctive features series. London: Routledge; 2010

24. Segal ZV, Williams JMG, Teasdale J. Mindfulness cognitive therapy for depression. A new approach to preventing relapse. New York: Guilford; 2002.

25. Kanas N. Group therapy for schizophrenic patients. Washington, DC: American Psychiatric Press; 1996.

26. Van den Brink E, Koster F. Mindfulness-based compassionate living. A new training programme to deepen mindfulness with heartfulness. London and New York: Routledge; 2015.

27. Beck, A.T., Rush, A.J, Shaw, B.F. \& Emery G. (1979): Cognitive Therapy of Depression. New York: Guilford Press.

28. Beck AT, Steer RA, Brown GK. Beck depression inventory: manual. San Anthonio: The Psychological Cooperation; 1996.

29. Beck AT, Steer RA, Ball R, Ranieri WF. Comparison of the Beck depression inventories-IA and II in psychiatric outpatients. J Pers Asses. 1996;67:588-97.

30. Beck AT, Steer RA. Manual for the Beck anxiety inventory. San Anthonio: The Psychological Cooperation; 1990

31. Rosenberg M. Society and the adolescent self-image. Princeton: Princeton University Press; 1965.

32. Robins, R.W, Hendin, H.M. \& Trzesniewski, K.H.(2001): Measuring global selfesteem: construct validation of a single-item measure and the Rosenberg self-esteem scale. Soc Pers Soc Psychol 27, 151-161.

33. Gilbert P. Compassion focused therapy: The CBT distinctive features series, vol. 6. London: Routledge; 2010. p. 43-51.

34. Gilbert, P. \& Choden. Mindful Compassion. Using the power of Mindfulness and Compassion to Transform our Lives. UK: Constable \& Robinson; 2013. Pp. 192-196.

35. Gilbert, P. \& Choden. Mindful Compassion. Using the power of Mindfulness and Compassion to Transform our Lives. UK: Constable \& Robinson; 2013. 9. pp 241-242.

36. Gilbert P. Compassion focused therapy: The CBT distinctive features series. London: Routledge; 2010. p. 129-36.

37. Gilbert, P. \& Choden. Mindful Compassion. Using the power of Mindfulness and Compassion to Transform our Lives. UK: Constable \& Robinson; 2013. Pp. 261-269.

38. Gilbert P. The compassionate mind. UK: Constable \& Robinson; 2009. p. 347-55.

39. Gillbert P. Compassion focused therapy: The CBT distinctive features series. London: Routledge; 2010. p. 130-6.

40. Gilbert, P. \& Choden. Mindful Compassion. Using the power of Mindfulness and Compassion to Transform our Lives. UK: Constable \& Robinson; 2013. pp. 275-280.

41. Gilbert P. Compassion focused therapy: The CBT distinctive features series. London: Routledge; 2010. p. 67-81.

42. Gilbert P. The compassionate mind. UK: Constable \& Robinson; 2009. p. 258-61.

43. Gilbert P. Compassion focused therapy: The CBT distinctive features series. London: Routledge; 2010. p. 199-207.

44. Gilbert P. Compassion focused therapy: The CBT distinctive features series. London: Routledge; 2010. p. 177-80.

45. Söchting, I. (2014): Cognitive Behavioral Group Therapy, Challenges and Opportunities. Wiley-Blackwell. UK. p. 100.

46. Yalom ID. The theory and practice of group therapy. New York: Basic Books; 1995.

\section{Submit your next manuscript to BioMed Central and we will help you at every step:}

- We accept pre-submission inquiries

- Our selector tool helps you to find the most relevant journal

- We provide round the clock customer support

- Convenient online submission

- Thorough peer review

- Inclusion in PubMed and all major indexing services

- Maximum visibility for your research

Submit your manuscript at www.biomedcentral.com/submit
Biomed Central 